Check for updates

Cite this: Chem. Sci., 2018, 9, 8121

๑ All publication charges for this article have been paid for by the Royal Society of Chemistry

Received 27th June 2018

Accepted 29th August 2018

DOI: $10.1039 / c 8 s c 02830 c$

rsc.li/chemical-science

\section{Anion exchange in inorganic perovskite nanocrystal polymer composites $\dagger$}

\author{
Maria Sygletou, (D) *a Maria-Eleni Kyriazi, ${ }^{\text {b }}$ Antonios G. Kanaras (D) b \\ and Emmanuel Stratakis (DD *ac
}

We demonstrate a facile, low-cost and room-temperature method of anion exchange in cesium lead bromide nanocrystals $\left(\mathrm{CsPbBr}_{3} \mathrm{NCs}\right)$, embedded into a polymer matrix. The anion exchange occurs upon exposure of the solid $\mathrm{CsPbBr}_{3} \mathrm{NCs} / \mathrm{PDMS}$ nanocomposite to a controlled anion precursor gas atmosphere. The rate and extent of the anion exchange reaction can be controlled via the variation of either the exposure time or the relative concentration of the anion precursor gas. Post-synthesis chemical transformation of perovskite nanocrystal-polymer composites is not readily achievable using conventional methods of anion exchange, which renders the gas-assisted strategy extremely useful. We envisage that this work will enable the development of solid-state perovskite NC optoelectronic devices.
Solution-processed all-inorganic cesium lead halide perovskite $\left(\mathrm{CsPbX}_{3}, \mathrm{X}=\mathrm{Cl}, \mathrm{Br}, \mathrm{I}\right)$ nanocrystals (NCs) have drawn a lot of attention lately, due to their exceptional optical properties, including medium optical bandgaps, strong absorption coefficients, high luminescence quantum yields and narrow emission bandwidths. ${ }^{1-5}$ Owing to these properties, they have been introduced as a new class of photoactive materials for nextgeneration, low-cost, high-performance flexible optoelectronics, ${ }^{6,7}$ including perovskite-based solar cells, ${ }^{8}$ lasing sources, ${ }^{9,10}$ photodetectors ${ }^{11}$ and light-emitting diodes ${ }^{12-15}$ with high brightness and tunable emission. At the same time, allinorganic perovskites exhibit higher thermal and chemical stability, ${ }^{16}$ as well as higher resistance to humidity ${ }^{17}$ than their organic-inorganic counterparts, such as $\mathrm{MAPbX}_{3}$. The stability of halide perovskite NCs still remains a research topic of great interest. ${ }^{18}$ It has been reported that the robustness of $\mathrm{CsPbX}_{3}$ NCs can be improved by the addition of a small amount of polymer (poly(maleic anhydride-alt-1-octadecene)-PMA into the precursor solutions, which creates an additional ligand coating around each individual NC, or via encapsulation into PMMA or polyethylene oxide). ${ }^{12,19-21}$ Furthermore, a silica-coating process

${ }^{a}$ Institute of Electronic Structure and Laser, Foundation for Research and Technology Hellas, Heraklion, 71110,Crete, Greece.E-mail: masyg@iesl.forth.gr; stratak@iesl. forth.gr

${ }^{b}$ Physics and Astronomy, Faculty of Physical Sciences and Engineering, University of Southampton, Southampton, SO171BJ, UK

'Department of Materials Science and Technology, University of Crete, Heraklion 71003, Crete, Greece

$\dagger$ Electronic supplementary information (ESI) available: Experimental details on the synthesis of the NCs, the preparation of the polymer:NCs nanocomposites and the anion exchange processes as well as optical and structural characterization of the nanocrystals and the nanocomposites are presented. See DOI: $10.1039 / \mathrm{c} 8 \mathrm{sc} 02830 \mathrm{c}$ has been reported to enhance the stability of inorganic perovskite NC-based LEDs. ${ }^{22,23}$ A prominent property of perovskite NCs is their ability to undergo a post-synthesis anion exchange, in solution, using chemical precursors or photo-induced processes. $^{24-27}$ Despite the numerous studies on anion exchange reactions in the liquid phase, only a few reports have demonstrated such reactions in solid state, either in the bulk or in the form of NCs. In particular, Hoffman $e t$ al. ${ }^{28}$ reported the conversion of $\mathrm{CsPBr}_{3}$ to $\mathrm{CsPbI}_{3}$ films following heat treatment with a $\mathrm{PbI}_{2}$ solution. While, Guhrenz et al. ${ }^{27,29}$ reported a method of anion exchange via the direct incorporation of $\mathrm{CsPbX}_{3}$ NCs into ion-rich matrices. In parallel, there have been reports of post-synthetic halide exchange reactions in organicinorganic metal-halide bulk perovskites (OIHPS) upon exposure to halogen $\left(\mathrm{X}_{2}\right)^{30-32}$ and hydrogen halide (HX) gases. ${ }^{33}$ Gasinduced formation/transformation (GIFT) of OIHPS has shown tremendous promise in various applications, including solar cells, optoelectronics, sensors, and beyond, however, a detailed understanding of the mechanisms underlying the GIFT phenomena is still lacking. ${ }^{31}$

In this communication, we introduce for the first time a GIFT process in perovskite NCs in solid state. In particular, we present a simple, post-synthesis and room temperature, solidstate anion exchange method to tune the emission properties of inorganic perovskite NCs, hosted into a polymer matrix. We demonstrate anion exchange in nanocomposite layers, comprising of $\mathrm{CsPbBr}_{3}$ NCs dispersed in polydimethylsiloxane (PDMS), upon their exposure to a halide precursor gas atmosphere at room temperature. Fig. 1 represents a schematic illustration of the schematic route followed for the transformation of $\mathrm{CsPbBr}_{3}$ to $\mathrm{CsPbCl}_{3}$. It is shown that the extent of the anion exchange reaction and therefore the NCs' emission properties can be finely tuned by adjusting the exposure time 


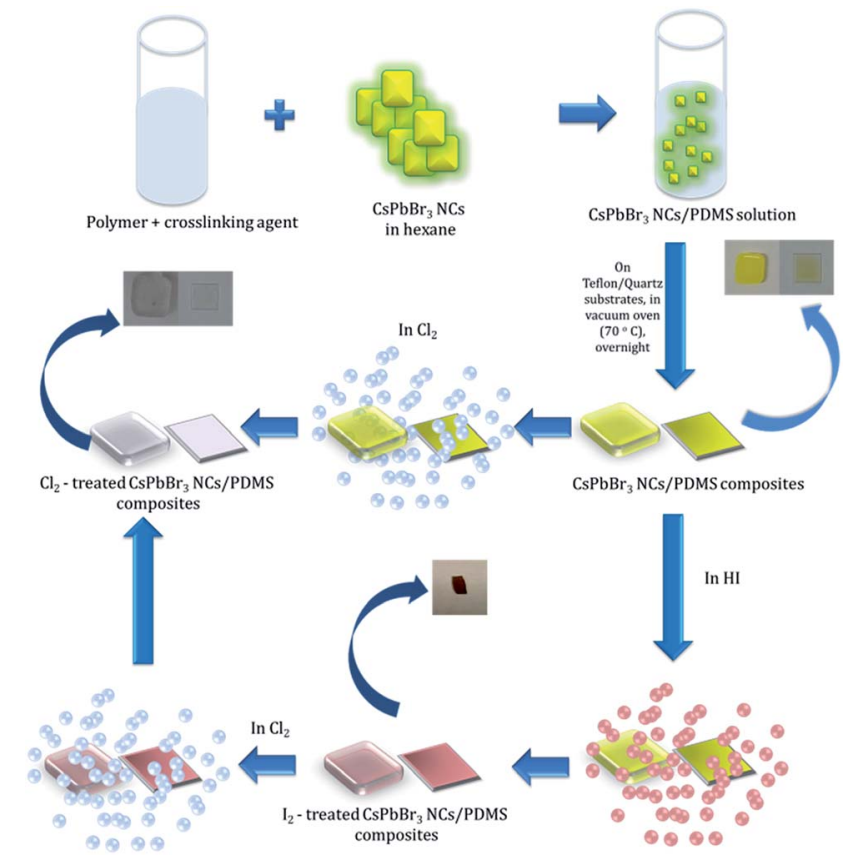

Fig. 1 Process flow of post-synthesis anion exchange in $\mathrm{CsPbBr}_{3} \mathrm{NCs}$ in solid phase due to $\mathrm{Cl}_{2}$ and/or $\mathrm{HI}$ treatment.

and concentration of $\mathrm{Cl}_{2}$ gas; the iodine anion exchange process is also demonstrated. Apart from the tunability of nanoparticle emission, it is shown that the PDMS matrix protects the NCs against adverse humidity effects, giving rise to stable optical properties. These properties can open up new avenues for the in situ and low-cost optical modulation of perovskite polymernanocomposites, useful in various optoelectronic applications.

Following synthesis, the NC colloids in hexane showed a characteristic fluorescence peak at $521 \mathrm{~nm}$, with a full width half maximum (FWHM) of $\sim 25 \mathrm{~nm}$ (Fig. S2 $\dagger$ ). The incorporation of NCs into PDMS $^{34}$ gave rise to a nanocomposite with a characteristic yellowish color under ambient light (Fig. 2, inset) and a pronounced green emission upon excitation with UV light (Fig. 3b). As shown in Fig. 2, the NCs' absorption

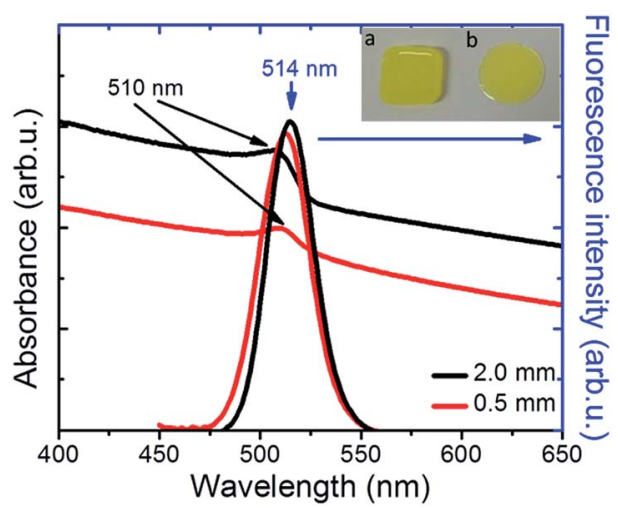

Fig. 2 Normalized UV-Vis absorption and fluorescence spectra of PDMS:NCs nanocomposites. The inset shows pictures of nanocomposites (a) formed on a Teflon mould (thickness of $2 \mathrm{~mm}$ ) and (b) drop-casted onto a quartz substrate (thickness of $0.5 \mathrm{~mm}$ ).
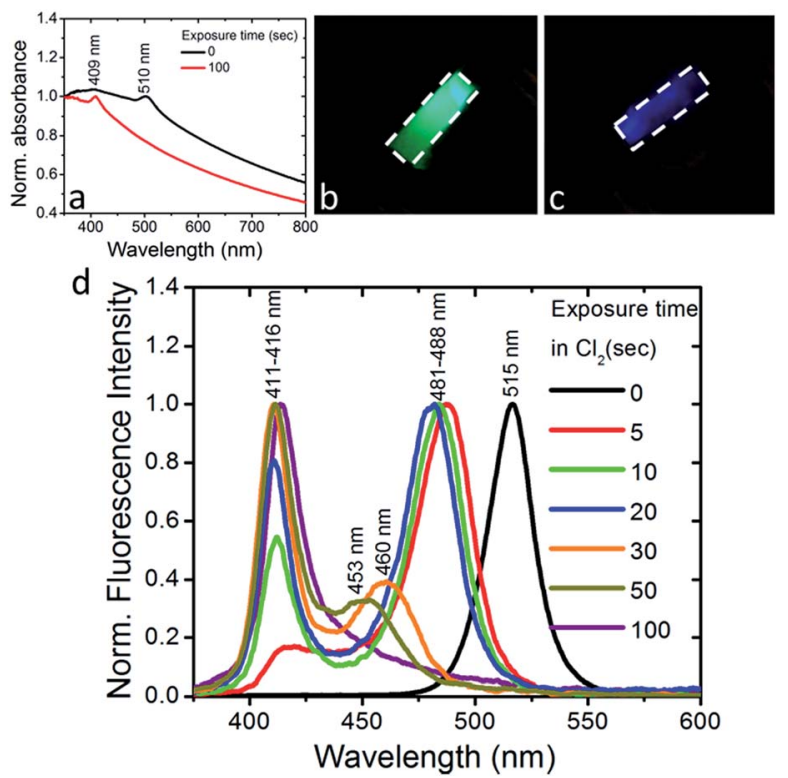

Fig. 3 (a) Normalized UV-Vis absorption spectra of PDMS: $\mathrm{CsPbBr}_{3} \mathrm{NC}$ nanocomposite layers of $0.5 \mathrm{~mm}$ thickness, before and after exposure to $\mathrm{Cl}_{2}$ gas with a partial pressure of 70 mbar for $100 \mathrm{~s}$. Images of a PDMS: $\mathrm{CsPbBr}_{3} \mathrm{NC}$ composite layer upon UV excitation, before (b) and after (c) exposure to $\mathrm{Cl}_{2}$ gas. The sample area is marked with the white dashed line. (d) Normalized fluorescence spectra of PDMS: $\mathrm{CsPbBr}_{3} \mathrm{NC}$ nanocomposite layers of thickness $0.5 \mathrm{~mm}$, before and after exposure to $\mathrm{Cl}_{2}$ gas for various time intervals.

maximum was slightly red-shifted from $495 \mathrm{~nm}$ in solution to $510 \mathrm{~nm}$ in the nanocomposite, while the fluorescence maximum was slightly blue-shifted from $521 \mathrm{~nm}$ in solution to $515 \mathrm{~nm}$ in the nanocomposite (Fig. 2). This is mainly due to the increase in the dielectric properties of the surrounding medium, from hexane with $n_{\text {hexane }}=2.06$, to PDMS with $n_{\text {PDMS }}$ $=2.3-2.8$. Furthermore, a slight broadening of the respective emission peak was observed due to the formation of NC clusters, which was by Two-Photon Excited Fluorescence (TPEF) Microscopy (Fig. S4†).

More importantly, the emission of NCs hosted into PDMS was observed to be remarkably stable over time, upon exposure to ambient conditions. This is in contrast to the widely reported sensitivity of $\mathrm{CsPbBr}_{3}$ NCs to ambient air and/or moisture. ${ }^{35-37}$ To further explore such emission stability and robustness against humidity, we investigated the emission spectra evolution of the PDMS: $\mathrm{CsPBBr}_{3}$ NC layers, following their immersion into water. It was observed that the prolonged $(24 \mathrm{~h})$ interaction of the nanocomposites with water caused no significant effect on their respective emission spectra (Fig. $\mathrm{S} 5 \dagger$ ). Furthermore the fluorescence spectra of the nanocomposites remain practically unchanged upon storage of the nanocomposites for 30 days in ambient conditions (Fig. S5†). Both of the above observations are strong indications that the polymer matrix successfully protects the NCs against the effects of humidity.

We also observed that the optical absorption and fluorescence spectra of the PDMS: $\mathrm{CsPBr}_{3}$ NC layers progressively blue-shifted upon their exposure to $\mathrm{Cl}_{2}$ gas, indicating the anionic exchange of the participating halides. The solid-state 
chlorination process is presented in the ESI. $\dagger$ Representative results are shown in Fig. 3. In particular, exposure to $\mathrm{Cl}_{2}$ gas, of 70 mbar-partial pressure, for $100 \mathrm{~s}$ gave rise to a blue-shift of both the absorption and emission peaks from $\sim 510 \mathrm{~nm}$ to $\sim 410 \mathrm{~nm}$. This shift is reasonable, considering that the emission peak of $\mathrm{CsPbBr}_{3} \mathrm{NCs}$ is around $510 \mathrm{~nm}$ while that of $\mathrm{CsPbCl}_{3} \mathrm{NCs}$ is observed at $\sim 390 \mathrm{~nm} \cdot{ }^{24}$ At the same time, quenching of the fluorescence quantum yield was observed. Both phenomena, i.e. the partial replacement of $\mathrm{Br}$ ions with $\mathrm{Cl}$ ions and the fluorescence quenching are in accordance with former findings $\mathrm{s}^{24,25}$ regarding NC colloids. It should be noted that the FWHM of the blue-emitting composite layers attained is comparable to that of the initial layers. In addition, an incomplete exchange reaction took place for the thickest $(\sim 2$ $\mathrm{mm}$ ) samples tested. This is presented in Fig. S6, $\dagger$ showing that two characteristic absorption peaks, at $\sim 409 \mathrm{~nm}$ and $\sim 465 \mathrm{~nm}$, arise upon exposure of the sample to a chlorine environment (Fig. S6 $†$ ). The corresponding fluorescence spectra confirm the emission from two peaks, at $\sim 411 \mathrm{~nm}$ and $\sim 475 \mathrm{~nm}$, with the latter being the most pronounced (Fig. S7 $†$ ). This is possibly due to the formation of mixed halide $\mathrm{CsPb}(\mathrm{Br} / \mathrm{Cl})$ NCs with different $\mathrm{Cl}: \mathrm{Br}$ ratios. On the contrary, in the case of a thinner layer $(\sim 500 \mu \mathrm{m})$, a single absorption peak at $409 \mathrm{~nm}$ is observed (Fig. 3a), while at the same time the emission peak shifts from $515 \mathrm{~nm}$ (Fig. 3d, black line) to $411 \mathrm{~nm}$ within $100 \mathrm{~s}$ of exposure to chlorine (Fig. 3d, violet line), indicating the formation of $\mathrm{CsPb}(\mathrm{Br} / \mathrm{Cl}) \mathrm{NCs}$ with a $\mathrm{Br}: \mathrm{Cl}$ ratio of $2: 3 .{ }^{24}$ Following the exposure for $100 \mathrm{~s}$, the phenomenon is partially reversible (Fig. S15†), i.e. the fluorescence spectrum slowly red-shifts with time and saturates to a peak emission value of $475 \mathrm{~nm}$, attributed to the chemical composition of $\mathrm{CsPbBr}_{3} \mathrm{Cl}_{2} \mathrm{NCs}(\mathrm{Br}: \mathrm{Cl}$ ratio of $3: 2$ ). In Fig. $3 \mathrm{~b}$ and c typical images of a nanocomposite layer under UV light excitation, before and after exposure to $\mathrm{Cl}_{2}$, are presented, respectively. It can be clearly seen that, the emitted green color of the pristine sample changes to blue upon chlorine treatment. Also, as shown in Fig. S8, $\dagger$ the color of the respective sample changes from yellow to light grey. In literature, anion conversion reactions have already been interpreted in terms of halogen reduction potentials, at least in the case of OIHPs. ${ }^{30}$ These studies showed that exposure of OIHPs to a halogen gas, $\mathrm{X}_{2}$, can displace the crystal halide anions, $\mathrm{Y}^{-}$, at room temperature, provided that $\mathrm{X}$ features a higher standard reduction potential than the displaced halide, $\mathrm{Y}^{30,31}$ Our results indicate that this could also occur in the all-inorganic lead halide perovskites as well. Considering the higher reduction potential of $\mathrm{Cl}_{2}$ compared to $\mathrm{Br}_{2}, \mathrm{Cl}_{2}$ can oxidize $\mathrm{Br}^{-}$and convert $\mathrm{CsPbBr}_{3}$ to $\mathrm{CsPbCl}_{3}$ with solely gas-phase by-products. In the case of PDMS: $\mathrm{CsPBr}_{3} \mathrm{NCs}$, this process is facilitated by the high permeability and diffusivity of $\mathrm{Cl}_{2}$ gas in PDMS, ${ }^{38}$ enabling chlorine atoms to interact with the embedded perovskite NCs. Based also on the relevant literature, the flow rate of $\mathrm{Cl}_{2}$ gas across a PDMS membrane is proportional to the difference in partial pressure and inversely proportional to the membrane thickness; ${ }^{39}$ this could account for the deficient anion exchange process taking place in the thicker nanocomposite layers.
To further shed light on the anion exchange process, the exposure of the nanocomposite layers to different $\mathrm{Cl}_{2}$ gas partial pressures was investigated. The corresponding results are presented in Fig. 4 and S9; $\dagger$ in these figures $I_{1}$ is the intensity of the initial emission peak $(\sim 515 \mathrm{~nm})$ and $I_{2}$ is the intensity of the emission peak that emerges upon exposure to $\mathrm{Cl}_{2}$ (i.e. at $\sim 435$ $\mathrm{nm}$ ). It can be observed (Fig. 4a) that, as the $\mathrm{Cl}_{2}$ gas pressure is increased from 0 to $70 \mathrm{mbar}$, the initial emission peak progressively blue-shifts and $I_{1}$ decreases, while, on the other hand, $I_{2}$ gradually increases. It is also shown in Fig. $4 \mathrm{~b}$ and c that both the $I_{2} / I_{1}$ intensity ratio and the $1^{\text {st }}$ emission peak shift tend to saturate at a similar $\mathrm{Cl}_{2}$ gas critical partial pressure $(\sim 20$ mbar). These observations indicate the potential of the perovskite nanocomposite layers to operate as halide gas sensing elements. It is notable that the fluorescence signal of these nanocomposites is preserved, even after $24 \mathrm{~h}$ of treatment with chlorine.

The photoluminescence quantum yield (PLQY) of the initial $\mathrm{CsPbBr}_{3}$ nanocrystals in hexane, measured via the comparative method, ${ }^{40}$ was equal to $48 \%$. Compared to the nanocrystals in solution, it is observed that when an equal vol\% of $\mathrm{CsPbBr}_{3}$ nanocrystals is embedded into PDMS, the photoluminescence intensity decreases (Fig. S16†). Accordingly, the corresponding PLQY measured for the PDMS:CsPbBr 3 NC layers was dropped to $36 \%$. Following chlorine treatment, the PLQY of the nanocomposites was decreased by almost 10 times, i.e. to $4 \%$, which is in accordance to previous reports on the anion exchange effect on the PLQY. ${ }^{24}$

Experiments in the presence of an iodine precursor gas were also performed, ${ }^{32}$ as schematically shown in Fig. 1 . The solid-

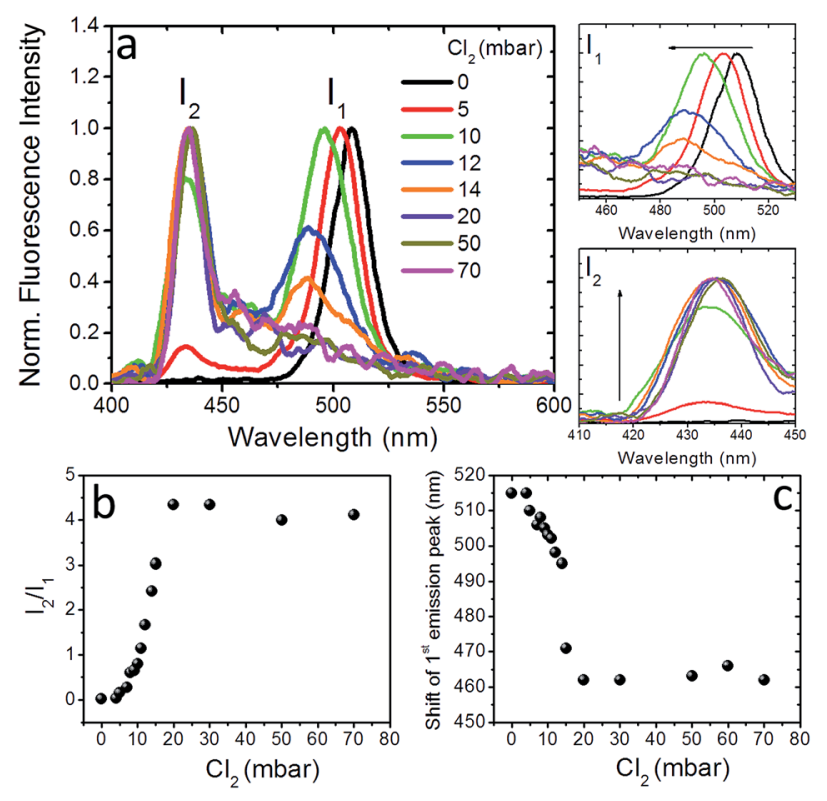

Fig. 4 (a) Normalized fluorescence spectra of, $0.5 \mathrm{~mm}$ thick, PDMS: $\mathrm{CsPbBr}_{3} \mathrm{NCs}$ nanocomposite layers upon exposure to different partial $\mathrm{Cl}_{2}$ pressures. The corresponding evolution of the initial peak with intensity $I_{1}$ (top) and of the peak that emerges after chlorine treatment with intensity $I_{2}$, (bottom) are shown on the right. (b) Fluorescence intensity ratio, $I_{2} / I_{1}$, and (c) spectral shift of the first emission peak, as a function of the partial pressure of $\mathrm{Cl}_{2}$ gas. 
state iodination process is presented in the ESI. $\uparrow$ Fig. 5a and $\mathrm{b}$ present the absorption and fluorescence spectra of the PDMS:CsPbBr ${ }_{3} \mathrm{NC}$ nanocomposite layers following sequential treatment, first with $\mathrm{I}_{2}$ gas under ambient conditions, followed by $\mathrm{Cl}_{2}$ gas. Following exposure to $\mathrm{I}_{2}$ gas under ambient conditions for 10 minutes, the nanocomposites showed a red-shifted emission peak at $\sim 660 \mathrm{~nm}(\sim 1.87 \mathrm{eV})$ that complies with that reported for $\mathrm{CsPbI}_{3} \mathrm{NCs}^{24}{ }^{24}$ Subsequently, these nanocomposites were placed in a chlorine environment and their emission peak was observed to blue-shift to $\sim 410 \mathrm{~nm}(3.02 \mathrm{eV})$, i.e. close to that observed upon direct chlorination of the pristine PDMS:CsPbBr ${ }_{3}$ NC layers. Considering the lower reduction potential of $\mathrm{I}_{2}$ compared to that of $\mathrm{Cl}_{2}$, a redox-type conversion reaction, i.e. oxidation of $\mathrm{Br}^{-}$by $\mathrm{I}_{2}$ and subsequent conversion of $\mathrm{CsPbBr}_{3}$ to $\mathrm{CsPbI}_{3}$, could not account for the observed displacement of the emission peak. However, it is well known that the ambient humidity remarkably affects the $I_{2}$ gas stability, leading to the formation of $\mathrm{HI}$ and HIO. ${ }^{\mathbf{4 1}}$ It has also been reported that mutual anion conversions in perovskite NCs can be alternatively realised upon exposure to gaseous $\mathrm{HX}$, via ion-exchange reactions. ${ }^{33}$ Based on this, the possibility of $\mathrm{HI}$ formation due to ambient humidity may account for the observed red shift in the UV-Vis and fluorescence spectra. Experiments involving exposure of $\mathrm{PDMS}: \mathrm{CsPbBr}_{3}$ NC nanocomposites to HX gases are currently in progress to clarify this issue.

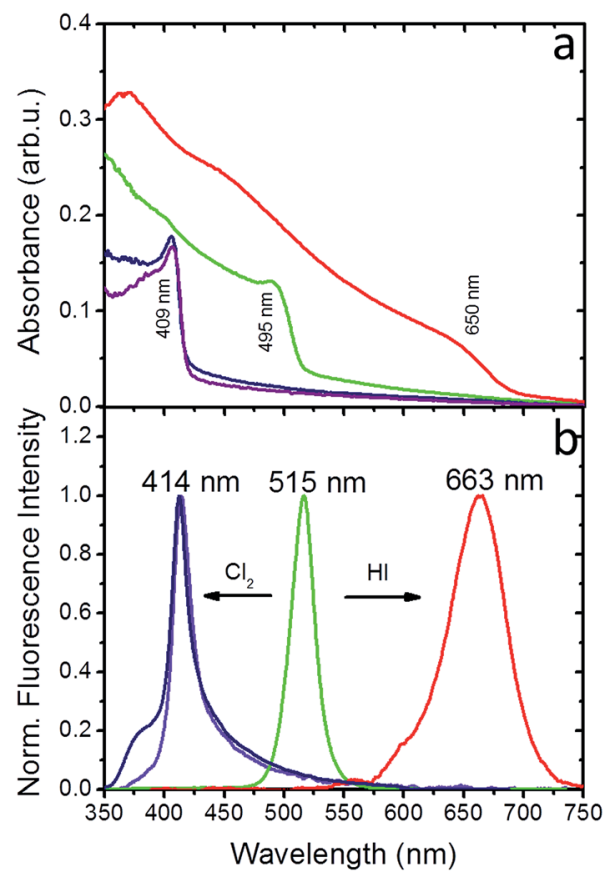

Fig. 5 (a) UV-Vis absorption of PDMS: $\mathrm{CsPbBr}_{3} \mathrm{NC}$ nanocomposite layer (green line), following $\mathrm{Cl}_{2}$ (purple line) and $\mathrm{HI}$ (red line) treatment for 10 minutes, as well as $\mathrm{Cl}_{2}$ treatment of the iodinated nanocomposite for 10 minutes (blue line). (b) Normalized fluorescence intensity of $\mathrm{PDMS}_{\mathrm{CsPbBr}} \mathrm{NC}$ nanocomposite layer (green line) following $\mathrm{Cl}_{2}$ (purple line) and $\mathrm{HI}$ (red line) treatment for 10 minutes, as well as $\mathrm{Cl}_{2}$ treatment of the iodinated nanocomposite for 10 minutes (blue line).
It can be concluded that the anion exchange process can only proceed along a single direction, that is $\mathrm{Br}^{-}>\mathrm{Cl}^{-}, \mathrm{Br}^{-}>\mathrm{I}^{-}, \mathrm{I}^{-}>$ $\mathrm{Cl}^{-}$. This is further confirmed by experiments with PDMS:CsPbI ${ }_{3}$ NCs nanocomposite layers showing a characteristic shift of the initial fluorescence peak to lower wavelengths upon exposure to $\mathrm{Cl}_{2}$ gas (Fig. S17 and S18†). Our findings comply with the reduction potential relationship of the three, considering that $\mathrm{Cl}_{2}$ exhibits higher reduction potential compared to $\mathrm{Br}_{2}$ and $\mathrm{I}_{2}$ exhibits higher reduction potential compared to $\mathrm{Br}_{2}$.

To further account for the microscopic mechanism behind the anion exchange process, FTIR, XPS and XRD spectra of the PDMS: $\mathrm{CsPbr}_{3}$ NC layers, prior and after chlorine treatment, were recorded. The corresponding FTIR spectra, presented in Fig. S10 and $\mathrm{S} 11, \uparrow$ reveal no significant change in the chemical structure of the nanocomposites following halogen gas treatment. The survey XPS scans (Fig. S12†), recorded from the samples before and after chlorine treatment, show mainly the presence of $\mathrm{O}, \mathrm{C}$ and $\mathrm{Si}$, attributed to the PDMS matrix.

Fig. $\mathrm{S} 13 \dagger$ shows the respective high-resolution XPS spectra of $\mathrm{Cs} 3 \mathrm{~d}, \mathrm{~Pb} 4 \mathrm{f}$ and $\mathrm{Br} 3 \mathrm{~d}$ peaks. Prior to $\mathrm{Cl}_{2}$ exposure, traces of $\mathrm{Cs}, \mathrm{Pb}$ and a small amount of $\mathrm{Br}$ were detected. While, after exposure to $\mathrm{Cl}_{2}$, traces of $\mathrm{Cs}, \mathrm{Pb}$ and a small amount of $\mathrm{Cl}$ were detected, indicating the replacement of $\mathrm{Br}$ with $\mathrm{Cl}$. Finally, the corresponding XRD spectra are presented in Fig. S14, $\dagger$ showing a shift of the characteristic peaks of $\mathrm{CsPbBr}_{3} \mathrm{NCs}$ from $29.05^{\circ}$ to $29.15^{\circ}$ and from $38.2^{\circ}$ to $39.2^{\circ}$, after chlorine treatment. On the contrary, exposure to $\mathrm{HI}$ gives rise to a shift of the NCs' XRD peaks to lower diffraction angles (Fig. S14 $\dagger$ ). Both of the above findings are in accordance to previous literature observations on $\mathrm{Br}-\mathrm{Cl}$ anion exchange reactions in perovskite NCs. ${ }^{24,25,42,43}$ In accordance to the emission spectra, the corresponding XRD spectra remain practically unaffected upon storage of the nanocomposites for 30 days in ambient conditions (Fig. S14†).

\section{Conclusions}

In summary, we have demonstrated a straightforward route to realize a, solid-state, anion exchange process in cesium lead halide perovskite NCs hosted into a polymer matrix. It is based on the exposure of perovskite NC:PDMS nanocomposite layers to a controlled halogen gas atmosphere. Using this method the nanocomposite absorption and emission properties can be spectrally tuned from the visible to ultraviolet, upon varying the exposure time to the respective halogen gas partial pressure. It is important to note here that the PDMS matrix constitutes a robust environment for the embedded perovskite NCs and secures their stability against humidity. The tunable optical characteristics, adjustable NC loadings and the ease of handling make the resulting nanocomposites attractive for applications in optoelectronics, e.g., as color conversion materials for solid-state lighting, laser gain media, and solar light concentrators. Most importantly, all inorganic cesium lead halide perovskite NC-based nanocomposites are presented as suitable candidates for halogen gas sensing applications. Presumably, the solid-state anion exchange strategy presented 
here can be practically applied to other inorganic as well as organic-inorganic perovskite polymer nanocomposites.

\section{Funding sources}

This work was supported by the State Scholarship Foundation (IKY) within the framework of the Action "Postdoctoral Researchers Support" (MIS: 5001552) from the resources of the OP "Human Resources Development, Education and Lifelong Learning" - ESPA 2014-2020 Program, contract number: 2016050-0503-8904.

\section{Conflicts of interest}

There are no conflicts to declare.

\section{Acknowledgements}

We acknowledge the technical assistance of Dr Sotiris Psilodimitrakopoulos for the TPEF images, Dr George Kenanakis for the FTIR measurements, Dr Labrini Sygellou for the XPS measurements and Ms Kyriaki Savva for the XRD analysis. E. S. acknowledges support of the European Research Infrastructure NFFA-Europe, funded by EU's H2020 Framework Program for research and innovation under grant agreement number No. 654360 .

\section{Notes and references}

1 L. Protesescu, S. Yakunin, M. I. Bodnarchuk, F. Krieg, R. Caputo, C. H. Hendon, R. X. Yang, A. Walsh and M. V. Kovalenko, Nano Lett., 2015, 15, 3692-3696.

2 X. Li, F. Cao, D. Yu, J. Chen, Z. Sun, Y. Shen, Y. Zhu, L. Wang, Y. Wei, Y. Wu and H. Zeng, Small, 2017, 1603996.

3 A. Kostopoulou, M. Sygletou, K. Brintakis, A. Lappas and E. Stratakis, Nanoscale, 2017, 9, 18202-18207.

4 K.-H. Wang, L. Wu, L. Li, H.-B. Yao, H.-S. Qian and S.-H. Yu, Angew. Chem., Int. Ed., 2016, 55, 8328-8332.

5 J. S. Yao, J. Ge, B. N. Han, K. H. Wang, H. Bin Yao, H. L. Yu, J. H. Li, B. S. Zhu, J. Z. Song, C. Chen, Q. Zhang, H. B. Zeng, Y. Luo and S. H. Yu, J. Am. Chem. Soc., 2018, 140, 3626-3634.

6 D. Liu, Q. Lin, Z. Zang, M. Wang, P. Wangyang, X. Tang, M. Zhou and W. Hu, ACS Appl. Mater. Interfaces, 2017, 9, 6171-6176.

7 J. Song, L. Xu, J. Li, J. Xue, Y. Dong and X. Li, Adv. Mater., 2016, 28, 4861-4869.

8 A. Kostopoulou, E. Kymakis and E. Stratakis, J. Mater. Chem. A, 2018, 6, 9765-9798.

9 Y. Wang, X. Li, J. Song, L. Xiao, H. Zeng and H. Sun, Adv. Mater., 2015, 27, 7101-7108.

$10 \mathrm{Y} . \mathrm{Fu}, \mathrm{H} . \mathrm{Zhu}, \mathrm{C}$. C. Stoumpos, Q. Ding, J. Wang, M. G. Kanatzidis, X. Zhu and S. Jin, ACS Nano, 2016, 10, 7963-7972.

11 P. Ramasamy, D.-H. Lim, B. Kim, S.-H. Lee, M.-S. Lee and J.-S. Lee, Chem. Commun., 2016, 52, 2067-2070.

12 M. Meyns, M. Peralvarez, A. Heuer-Jungemann, W. Hertog, M. Ibanez, R. Nafria, A. Genc, J. Arbiol, M. V. Kovalenko,
J. Carreras, A. Cabot and A. G. Kanaras, ACS Appl. Mater. Interfaces, 2016, 8, 19579-19586.

13 F. Palazon, F. Di Stasio, Q. A. Akkerman, R. Krahne, M. Prato and L. Manna, Chem. Mater., 2016, 28, 2902-2906.

14 X. Li, Y. Wu, S. Zhang, B. Cai, Y. Gu, J. Song and H. Zeng, Adv. Funct. Mater., 2016, 26, 2435-2445.

15 P. Fu, Q. Shan, Y. Shang, J. Song, H. Zeng, Z. Ning and J. Gong, Sci. Bull., 2017, 62, 369-380.

16 T. Xu, L. Chen, Z. Guo and T. Ma, Phys. Chem. Chem. Phys., 2016, 18, 27026-27050.

17 J. Liang, C. Wang, Y. Wang, Z. Xu, Z. Lu, Y. Ma, H. Zhu, Y. Hu, C. Xiao, X. Yi, G. Zhu, H. Lv, L. Ma, T. Chen, Z. Tie, Z. Jin and J. Liu, J. Am. Chem. Soc., 2016, 138, 15829-15832.

18 H. Huang, M. I. Bodnarchuk, S. V. Kershaw, M. V. Kovalenko and A. L. Rogach, ACS Energy Lett., 2017, 2, 2071-2083.

19 K. Ma, X.-Y. Du, Y.-W. Zhang and S. Chen, J. Mater. Chem. C, 2017, 5, 9398-9404.

20 S. N. Raja, Y. Bekenstein, M. A. Koc, S. Fischer, D. Zhang, L. Lin, R. O. Ritchie, P. Yang and A. P. Alivisatos, ACS Appl. Mater. Interfaces, 2016, 8, 35523-35533.

21 Y. Wang, J. He, H. Chen, J. Chen, R. Zhu, P. Ma, A. Towers, Y. Lin, A. J. Gesquiere, S. T. Wu and Y. Dong, Adv. Mater., 2016, 28, 10710-10717.

22 D. N. Dirin, L. Protesescu, D. Trummer, I. V. Kochetygov, S. Yakunin, F. Krumeich, N. P. Stadie and M. V. Kovalenko, Nano Lett., 2016, 16, 5866-5874.

23 C. Sun, Y. Zhang, C. Ruan, C. Yin, X. Wang, Y. Wang and W. W. Yu, Adv. Mater., 2016, 28, 10088-10094.

24 Q. A. Akkerman, V. D. Innocenzo, S. Accornero, A. Scarpellini, A. Petrozza, M. Prato and L. Manna, J. Am. Chem. Soc., 2015, 137, 10276-10281.

25 D. Parobek, Y. Dong, T. Qiao, D. Rossi and D. H. Son, J. Am. Chem. Soc., 2017, 139, 4358-4361.

26 G. Nedelcu, L. Protesescu, S. Yakunin, M. I. Bodnarchuk, M. J. Grotevent and M. V. Kovalenko, Nano Lett., 2015, 15, 5635-5640.

27 C. Guhrenz, A. Benad, C. Ziegler, D. Haubold, N. Gaponik and A. Eychmuller, Chem. Mater., 2016, 28, 9033-9040.

28 J. B. Hoffman, A. L. Schleper and P. V. Kamat, J. Am. Chem. Soc., 2016, 138, 8603-8611.

29 A. Benad, C. Guhrenz, C. Bauer, F. Eichler, M. Adam, C. Ziegler, N. Gaponik and A. Eychmueller, ACS Appl. Mater. Interfaces, 2016, 8, 21570-21575.

30 D. Solis-Ibarra, I. C. Smith and H. I. Karunadasa, Chem. Sci., 2015, 6, 4054-4059.

31 Y. Zhou and N. P. Padture, ACS Energy Lett., 2017, 2, 2166-2176. 32 D. Solis-Ibarra and H. I. Karunadasa, Angew. Chem., Int. Ed., 2014, 53, 1039-1042.

33 K. Chen, X. Deng, R. Goddard and H. Tüysüz, Chem. Mater., 2016, 28, 5530-5537.

34 I. D. Johnston, D. K. McCluskey, C. K. L. Tan and M. C. Tracey, J. Micromech. Microeng., 2014, 24, 35017.

35 S. Huang, Z. Li, B. Wang, N. Zhu, C. Zhang, L. Kong, Q. Zhang, A. Shan and L. Li, ACS Appl. Mater. Interfaces, 2017, 9, 7249-7258.

36 Q. Shan, J. Li, J. Song, Y. Zou, L. Xu, J. Xue, Y. Dong, C. Huo, J. Chen, B. Han and H. Zeng, J. Mater. Chem. C, 2017, 5, 4565-4570. 
37 T. Leijtens, G. E. Eperon, N. K. Noel, S. N. Habisreutinger, A. Petrozza and H. J. Snaith, Adv. Energy Mater., 2015, 5, 1500963.

38 M.-B. Hägg, J. Membr. Sci., 2000, 170, 173-190.

39 M. S. Eikeland, M. B. Hägg, M. A. Brook, M. Ottøy and A. Lindbråthen, J. Appl. Polym. Sci., 2002, 85, 2458-2470.

40 M. Grabolle, M. Spieles, V. Lesnyak, N. Gaponik, A. Eychmüller and U. Resch-Genger, Anal. Chem., 2009, 81, 6285-6294.
41 I. Lengyel, J. Li, K. Kustin and I. R. Epstein, J. Am. Chem. Soc., 1996, 118, 3708-3719.

42 G. Nedelcu, L. Protesescu, S. Yakunin, M. I. Bodnarchuk, M. J. Grotevent and M. V. Kovalenko, Nano Lett., 2015, 15, 5635-5640.

43 D. Zhang, Y. Yang, Y. Bekenstein, Y. Yu, N. A. Gibson, A. B. Wong, S. W. Eaton, N. Kornienko, Q. Kong, M. Lai, A. P. Alivisatos, S. R. Leone and P. Yang, J. Am. Chem. Soc., 2016, 138, 7236-7239. 